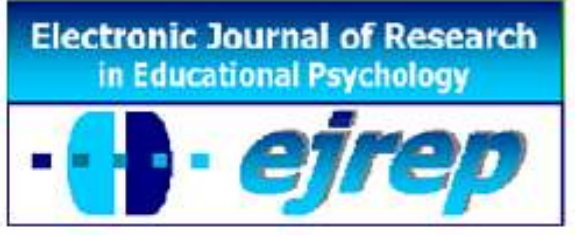

\title{
El desarrollo de la capacidad de Resiliencia Matemática: La voz de los estudiantes sobre el uso de las TIC en la aula
}

\author{
Ana Isabel Mota, Hélia Oliveira y Ana Henriques
}

Instituto de Educación, Universidad de Lisboa, Lisboa

\section{Portugal}

Correspondencia: Hélia Oliveira. Instituto de Educação da Universidade de Lisboa, Alameda da Universidade, 1649-013 Lisboa. Portugal. E-mail: hmoliveira@ie.ulisboa.pt

(C) Education \& Psychology I+D+i and Ilustre Colegio Oficial de la Psicología de Andalucía Oriental (Spain) 


\section{Resumen}

Introducción. La resiliencia matemática se asume como uno de los temas más importantes en el contexto escolar y la atención al respecto ha de ser prioritaria, dada la angustia exhibida por los estudiantes en el aprendizaje y las dificultades de comprensión de los conocimientos básicos en matemáticas año tras año. El objetivo principal de esta investigación fue el estudio de cómo los estudiantes que asisten a escuelas intermedias en el área suburbana de la capital portuguesa son capaces de reconocer y expresar las características de resiliencia matemática después de resolver varias tareas en grupos utilizando las TIC.

Método. La investigación se realizó con 64 estudiantes del octavo grado, de tres escuelas en desventaja socioeconómica, que respondieron a un cuestionario breve de composición abierta sobre su experiencia en el trabajo en una secuencia de tres tareas desarrolladas en el aula de matemáticas, con el uso de las TIC. Diferentes categorías y subcategorías han surgido a través del análisis de contenido inductivo realizado.

Resultados. Los resultados sugieren que los estudiantes perciben la experiencia del uso de las TIC en sus clases de matemáticas como útiles para su proceso de aprendizaje y que apreciaban a resolver tareas a través de un entorno de enseñanza-aprendizaje práctico. Los estudiantes también destacaron la importancia del apoyo de sus pares y del software TinkerPlots, percibido como una herramienta útil para mejorar su comprensión de conceptos matemáticos y el aprendizaje y que ha propiciado que esta experiencia de aprendizaje resultara más interesante y accesible para ellos. Sin embargo, un número residual de los estudiantes manifestó sentimientos de angustia, mientras que otros de indiferencia.

Discusión y Conclusión. Los resultados de esta investigación sugieren que estos alumnos presentan algunas características que la literatura especializada indica como típicas de los estudiantes con resiliencia matemática. Se exponen algunas implicaciones para agentes educativos, redundando en la necesidad de más investigaciones para ampliar la comprensión de cómo las herramientas TIC en ciertos ambientes pueden ser eficaces para mejorar el aprendizage de los estudiantes mientras promueven conductas resilientes en las matemáticas.

Palabras Clave: Resiliencia matemática, las ICT, TinkerPlots, Voz de los estudiantes, Aprendizaje 


\title{
Developing Mathematical Resilience: Students' Voice About the Use of ICT in Classroom
}

\begin{abstract}
Introduction. Mathematical resilience is assumed as one of the most important areas in school context and whose focus should be given priority, due to the distress exhibited by students when learning and understanding basic knowledge in mathematics year after year. The main goal of this research was to study how students attending middle schools in the suburban area of the Portuguese capital are able to recognize and express mathematical resilience characteristics after solving several tasks in groups using ICT.
\end{abstract}

Method. This research was conducted with 64 students from the $8^{\text {th }}$ grade, from three socioeconomic disadvantaged schools, who answered a short open-ended questionnaire regarding their experience in working on a sequence of three articulated tasks in the mathematics classroom, with the use of ICT. Different categories and subcategories have emerged from the conducted inductive content analysis.

Results. The results of this study suggest students perceived the experience of using ICT in their mathematics lessons as useful for their learning process and appreciated to enroll tasks through a practical teaching-learning environment. Students also highlighted the importance of their peers' support and perceived TinkerPlots software as a useful tool to enhance their understanding and learning of mathematical concepts, pointing how this learning experience became more interesting and easy to them. Nevertheless, a residual number of students reported feelings of distress whilst others indifference.

Discussion and Conclusion. The results of this research suggest these students exhibit some characteristics the specialized literature has pointed as typical from mathematical resilient students. Some implications for school practitioners are formulated but further research is needed to enlarge the understanding of how ICT tools in certain environments can be effective to enhance students' learning while promoting mathematical resilient behaviors.

Palabras Clave: Mathematical resilience, ICT, TinkerPlots, Students' voice, Learning 


\section{Introducción}

La resiliencia es una construcción clásica de la Psicología de la Educación y ha sido el foco principal de numerosas investigaciones en las últimas diez décadas (p.e., Luthar, 2007). La resiliencia se puede definir como "cualquier comportamiento, de atribución, o respuesta emocional a un desafío académico o social que es positivo y beneficioso para el desarrollo" (Yeager \& Dweck, 2012, p.303).

A pesar de que la resiliencia se ha estudiado de forma exhaustiva con los niños y jóvenes que viven en entornos de riesgo (p.e., drogas, violencia, pobreza), es escasa la investigación desarrollada con los niños y jóvenes que viven en contextos normativos. Consideramos que, si la comprensión de cómo los niños y jóvenes que viven en entornos de riesgo es fundamental para ayudar a desarrollar el éxito a pesar de la adversidad que enfrentan en su vida diaria, es también importante ampliar el conocimiento y la comprensión de cómo ayudar jóvenes de contextos normativos para desarrollar itinerarios educativos exitosos.

Dada la naturaleza multidimensional y compleja de la resiliencia resulta importante analizar y comprender esta construcción en contextos específicos (Luthar, 2007). La resiliencia matemática se asume como una de las variables centrales en el contexto escolar y a cuya atención se debe dar prioridad, teniendo en cuenta la dificultad presentada por los estudiantes en el aprendizaje de la comprensión y el conocimiento matemático básico año tras año. En Portugal, las matemáticas es una de las áreas más temidas y en la que el rendimiento académico es más problemático. Como sucede en nuestro contexto nacional, la estadística es un dominio en la materia de matemáticas para todos los grados desde la educación primaria a la educación secundaria en muchos países y, por lo tanto, los estudiantes la conciben como uno de los temas que tienen que aprender en matemáticas. Como este tema se enseña a menudo simplemente como una lista de gráficos y procedimientos para su construcción no considerándose su naturaleza específica, hemos desarrollado un proyecto con el fin de ayudar a los estudiantes a comprender el propósito y la utilidad de los datos en contextos de la vida real, proponiendo tareas más exigentes con las Tecnologías de la Información y Comunicación (TIC; Henriques \& Oliveira, 2016).

Esta investigación se desarrolló en el marco de un proyecto anterior y se centra en el dominio particular de la capacidad de resiliencia matemática. Su principal objetivo es enten- 
der cómo los estudiantes de secundaria son capaces de reconocer y exhibir características de resiliencia matemática después de resolver tareas utilizando un software específico en el tema de la estadística. Esta investigación supone un primer acercamiento a la investigación desarrollada en todo el mundo con respecto a este tema, llevado a cabo en el ámbito de la educación estadísticacon los estudiantes portugueses de secundaria provenientes de contextos escolares desfavorecidos socioeconómicamente que representan los itinerarios educativos normativos en escuelas suburbanas en la zona de Lisboa

\section{Resiliencia Matemática}

La matemática es percibida por los estudiantes como una de las materias escolares más exigentes y problemáticas y en la que, muy a menudo, el rendimiento académico se ve comprometido desde al comienzo de cada año académico. Los estudiantes pueden tener dificultades para las matemáticas debido a varias razones y en diferentes conceptos, siendo también diversas las estrategias que cada uno adopta para hacer frente a las mismas (González-Torres \& Artuch-Regarde, 2014; Lee \& Johnston-Wilder, 2013). ¿Cómo podemos entonces distinguir los estudiantes que presentan ansiedad cuando se enfrentan a situaciones exigentes de razonamiento matemático y evitan o se niegan a participar en esas tareas, de los que consideran un problema matemático como un reto y como medio de desarrollo de sus habilidades y conocimientos? La resiliencia matemática es el constructo psicológico que puede explicar mejor los comportamientos que los individuos adoptan cuando se enfrentan a situaciones difíciles y menos favorables y, sin embargo, alcanzan el éxito (Lee \& Johnston-Wilder, 2013).

La resiliencia matemática asume un enfoque de la psicología positiva e implica a aquellos que son capaces de superar los obstáculos afectivos en su proceso de aprendizaje. Los estudiantes que están matemáticamente resilientes se adaptan fácilmente y son capaces de hacer frente a situaciones difíciles y ambiguas a pesar de las barreras con las que se encuentran (Lee \& Johnston-Wilder, 2013). Estos estudiantes tienen una mentalidad de crecimiento, ya que saben que tienen la fuerza necesaria para superar las adversidades académicas y no académicas. Tener una mentalidad de crecimiento significa que los estudiantes son capaces de tomar decisiones con autonomíaen el proceso de aprendizaje, de experimentarse a sí mismos como parte de la comunidad escolar, y de percibirse a sí mismos como parte del proceso de aprendizaje (Dweck, 2000). En estos tipos de clases, los estudiantes tienden a sentirse seguros y perseveran incluso cuando se enfrentan a dificultades a medida que adquieren habilidades 
de comunicación y vocabulario para expresar sus interpretaciones matemáticas (Yeager \& Dweck, 2012).

De acuerdo con Lee y Johnston-Wilder (2014), los estudiantes que son matemáticamente resilientes (i) creen que la capacidad de aprender y dominar los conceptos matemáticos no es estática y limitada a unos pocos individuos, y que cualquiera puede ampliar sus habilidades y convertirse en mejores resultados en matemáticas; (ii) entienden la instrumentalidad de las matemáticas, es decir, reconocen su valor en su vida diaria dentro y fuera del contexto académico; (iii) entienden que, para el desarrollo de conceptos matemáticos, se requiere esfuerzo, perseverancia y curiosidad; y (iv) reconocen la importancia de los otros significativos en el apoyo que necesitan, tales como profesores o sus compañeros, así como herramientas como las TIC.

Investigaciones anteriores sugieren la importancia de promover intencionadamente la resiliencia matemática dentro de las aulas para mejorar el rendimiento de los estudiantes (p.e., Johnston-Wilder \& Lee, 2010; Yeager \& Dweck, 2012); se necesita de esta intencionalidad por parte de todos los estudiantes, pero especialmente por parte de los que presentan mayor angustia y están en riesgo de fracaso académico (Rivera \& Waxman, 2011).

En este trabajo, nos basamos en la voz de los estudiantes como punto de partida para entender cómo la resiliencia matemática podría ser mejorada y, en consecuencia, cómo el aprendizaje de matemáticas podría facilitarse. La voz de los estudiantes ha sido reconocida como una poderosa fuente de información sobre temas educativos, ya que ofrece una visión de gran alcance para los profesores en relación con las experiencias de los estudiantes y oportunidades de aprendizaje para la mejora del aprendizaje efectivo de los alumnos (p.e., Deaney, Ruthven, \& Hennessy, 2003; Flutter, 2007; Lee \& Johnston-Wilder, 2013). Mientras los profesores se dan cuenta de lo que sienten y piensan los estudiantes mientras aprenden, promueven oportunidades a los estudiantes para asumir la responsabilidad en su propio proceso de aprendizaje (Flutter, 2007).

\section{Tecnologías de la Información y Comunicacion (TIC)}

En la última década se han realizado varios estudios sobre el uso de las TIC en la enseñanza y el aprendizaje en las clases de matemáticas (p.e., Fitzallen \& Watson, 2010). Aunque el impacto de las TIC en la resiliencia matemática de los estudiantes no ha sido siempre como se esperaba (Lugalia, Johnston-Wilder, \& Goodall, 2003), la mayor parte de las investi- 
gaciones señala el uso de estas herramientas como eficaz en la promoción de las conductas resilientes de los estudiantes (Livingstone, 2012), ya que refuerzan la participación en el aula, la motivación para aprender y el rendimiento en lo desarrollo de las tareas escolares (Deaney et al., 2003).

A pesar de la reconocida importancia de las TIC en el proceso de enseñanzaaprendizaje, hay que hacer notar que las TIC per se no aumentan o mejoran la calidad de ese proceso (Sutherland et al., 2004). Las teorías sobre la resiliencia se basan en un modelo ecológico y ponen de relieve la importancia del contexto al que el individuo pertenece y opera (Fergus \& Zimmerman, 2005). De hecho, es importante tener en cuenta las influencias socioculturales de los conocimientos previos que los profesores y estudiantes aportan a cada nueva situación de aprendizaje, puesto que ello determinará la forma en que se desarrollará el nuevo conocimiento (Kent \& Cortadora, 2004). Esta premisa refuerza el papel central del profesor en el modo en que se introdujeron las actividades matemáticas con las TIC, para asegurar que todos los estudiantes pueden aprender significativamente (Deaney et al., 2003). Como Moore (1997) señaló, “el cambio sustancial en la enseñanza de la estadística se basa en una fuerte sinergia entre el contenido, la pedagogía y la tecnología” (p.123).

En el contexto de la educación estadística, algunos autores sostienen la necesidad de crear un Ambiente de Aprendizaje del Raciocinio Estadístico (Statistical Reasoning Learning Environment [SRLE]), donde las tareas propuestas a los estudiantes tienen algunos principios subyacentes (Cobb \& McClain, 2004; Garfield \& Ben-Zvi, 2009). Los principios SRLE postulan que un ambiente de aprendizaje favorecido se produce cuando las actividades del aula están diseñadas para estimular el razonamiento y el discurso de los estudiantes mediante el uso de datos reales y motivadores para la ideas centrales, al mismo tiempo que se utilizan herramientas tecnológicas adecuadas y sistemas de evaluación diferentes (p.e., Garfield \& Ben-Zvi, 2009).

El software TinkerPlots es una de las herramientas tecnológicas utilizadas en este tipo de ambientes de aprendizaje. Fue diseñado para ayudar a los estudiantes en la exploración de datos y las investigaciones estadísticas. Su visualización de datos y herramientas de modelado se pueden utilizar desde los grados elementales, ya que muchas de estas herramientas son independientes de contenido específico presente en el programa escolar. Utilizando una multiplicidad de representaciones gráficas con TinkerPlots, los estudiantes pueden crear gráficos 
non estándar y aprender a interpretar los datos de una manera intuitiva y, por lo tanto, organizar gradualmente los datos para responder a sus preguntas (Konold \& Miller, 2005).

Este software fue lanzado en 2004 y ha sido progresivamente actualizado a partir de los resultados de investigaciónes llevadas a cabo al respecto. Varios estudios sobre su uso en el aula han evidenciado resultados positivos y se ha demostrado que es una herramienta útil para muchos profesores en todo el mundo (p.e., Ben-Zvi, 2006; Fitzallen, 2007; Garfield, Delmas, \& Zieffler, 2012; Henriques \& Oliveira 2016; Makar, Bakker, \& Ben-Zvi, 2011; Watson, 2012).

\section{Objetivos}

El objetivo de esta investigación es estudiar cómo los estudiantes de secundaria de la zona suburbana de la capital portuguesa son capaces de reconocer y expresar las características de resiliencia matemática después de resolver varias tareas en grupo utilizando las TIC.

\section{Método}

\section{Participantes}

Los participantes fueron 64 estudiantes, 31 chicas y 33 chicos, de entre 13 y 16 años de edad, pertenecientes al $8^{\circ}$ grado de tres escuelas públicas portuguesas en la zona de Lisboa. Estas escuelas se localizan en áreas suburbanas con condiciones socioeconómicas desfavorecidas y donde prevalecen múltiples etnias; varios de estos estudiantes provienen de familias monoparentales y tienen antecedentes de retención escolar. En estas escuelas, se estima que al menos el $40 \%$ de los estudiantes se benefician del apoyo financiero social para las comidas y los libros de texto y otros materiales escolares. Ninguno de estos estudiantes ha tenido experiencias previas con las TIC en sus clases de matemáticas.

\section{Instrumentos}

Un breve cuestionario abierto fue desarrollado para acceder al feedback de los estudiantes en relación con las actividades que se inscribieron en las clases utilizando el software TinkerPlots (versión 2.2). En este cuestionario se preguntaba a los estudiantes sobre qué tareas eran las que más habían valorado (datos que no son objeto de análisis del presente trabajo) así se proponían cuatro preguntas planteadas tanto en forma positiva como negativa. Por ello razón, en este trabajo asumimos que los estudiantes respondieron a un total de ocho preguntas. Las cuatro preguntas planteadas en una forma positiva fueron: (i) “¿En tu opinión, el uso 
del TinkerPlots para desarrollar las tareas facilitó tu aprendizaje estadística? Si es así, explica por qué.”; (ii) “¿Cuáles fueron las ventajas de utilizar TinkerPlots en el clase?”; (iii) “¿Qué has disfrutado más al trabajar con TinkerPlots?”; y (iv) “¿Te ayudó el trabajo en grupo para desarrollar las tareas? Explica". Las cuatro preguntas planteadas en una forma negativa fueron: (v) “¿En tu opinión, el uso de TinkerPlots para desarrollar las tareas no facilitó el aprendizaje estadística? Si es así, explica por qué.”; (vi) “¿Cuáles fueron las desventajas de usar TinkerPlots en el clase?”; (vii) “¿Qué has disfrutado menos en el trabajo con TinkerPlots?”; y (viii) “¿El trabajo en grupo no te ayudó a desarrolar las tareas? Explica”.

\section{Procedimiento}

Los datos fueron recogidos en el ámbito de un proyecto de investigación y desarrollo titulado "Promoción del Raciocinio Estadístico en la Educación Básica Utilizando la Tecnología”, organizado por las autoras en el Instituto de Educación de la Universidad de Lisboa. Este proyecto fue desarrollado con profesores de matemáticas de Educación Primaria y Secundaria y consistió en la planificación y ejecución de secuencias de tareas de raciocinio estadístico en clase. Los estudiantes trabajaron en grupos de tres o cuatro y utilizaran el software TinkerPlots (Versión 2.2) para resolver las tareas.

Estas tareas siguen los principios básicos de Cobb y McClain (2004) para el diseño de las secuencias de tareas y lo ambiente de las lecciones fue basado en los principios SRLE (Garfield \& Ben-Zvi, 2009; Henriques \& Oliveira, 2016) con el objetivo subyacente principal de ayudar a los estudiantes a desarrollar una cultura estadística y pensar y razonar sobre la información estadística.

Tres tareas articuladas se han implementado en estas aulas, a saber: (i) Nenana Ice Classic, donde se pidió a los estudiantes que analizaran diferentes tipos de representaciones e hicieran predicciones basadas en datos reales disponibles en la base de datos TinkerPlots; (ii) Una Experiencia com Peces, donde los estudiantes han participado en el proceso de toma de decisiones mediante la comparación de distribuciones por medio de simulaciones proporcionadas por la herramienta TinkerPlots; y (iii) El cuerpo humano, una investigación estadística para descubrir más acerca de los estudiantes en su escuela, donde se iniciaron mediante la toma de nota de sus medidas y luego hicieron juicios y predicciones informales (Henriques \& Oliveira, 2016). 
Hacia el final de la experiencia, se les pidió a los estudiantes que respondieran individualmente a un breve cuestionario abierto desarrollado para esta investigación.

\section{Análisis de datos}

Se basa en un análisis de contenido inductivo de acuerdo a las tres etapas sugeridas por Bardin (2014), a saber: (i) pre-análisis, que se caracteriza por la primera lectura de las respuestas dadas por los estudiantes con el objetivo de organizar la información y llegar a una comprensión más profunda y familiarización con el material escrito; (ii) la exploración, que se caracteriza por la categorización de los datos originales en unidades de contexto y registro que son mutuamente excluyentes, homogéneas, pertinentes, objetivas, fiables y productivas; y (iii) el tratamiento de datos, inferencia e interpretación, validado por tres investigadores que han llegado a un acuerdo de forma independiente con respecto a la etapa de exploración y siguientes.

A través del análisis de contenido surgieron diferentes categorías principales, cada una organizada en varias subcategorías. Las preguntas planteadas en forma positiva fueron de las que se obtuvo lo mayor aporte de los estudiantes y se originaron tres categorías principales y diez subcategorías.

La categoría de la Clase se centra en las cuestiones relacionadas con la transformación del ambiente del aula llevada a cabo por este experimento. Dos subcategorías fueron identificadas en esta categoría: Novedad y Grupos. La Novedad está presente cuando los estudiantes expresan que disfrutaron y valoran la oportunidad de aprender en un ambiente no tradicional, diferente a lo que están acostumbrados. La de Grupos se refiere a cómo los estudiantes han apreciado el trabajo con sus compañeros para la finalización de las tareas y cómo valoraban la ayuda mutua realizada por grupos, discutir con los compañeros y llegar a conocer las opiniones de los otros, ayudados y motivados en el proceso de aprendizaje.

La categoría de Tecnología se centra en las cuestiones directamente relacionadas con la inclusión de las TIC en las clases de matemáticas, donde los libros y ejercicios usando lápiz y papel fueron reemplazados por ordenadores y la exploración del TinkerPlots. Cuatro subcategorías surgieron: Experiencia, Organización, Práctico y Medidas. La Experiencia respecta a los estudiantes que experimentan el TinkerPlots como software para aprender matemáticas. Organización se refiere a la percepción que los estudiantes tienen de las ventajas de Tinker- 
Plots en la adecuación de la síntesis y organización de los datos sensibles y en la visualización y el manejo intuitivo de la gran cantidad de información que resulta de los datos. La subcategoria Práctico se relaciona con el carácter práctico del trabajo con TinkerPlots y cómo los estudiantes disfrutaron y valoraron el hecho de poder manipular los datos para desarrollar y hacer cálculos de una manera más rápida, simple y directa. La subcategoría Medidas se refiere a las referencias de los estudiantes acerca de las ventajas de TinkerPlots en el análisis de las medidas estadísticas específicas, tales como la media y la mediana, un importante objetivo de aprendizaje estadístico en este grado escolar.

La categoría de Aprendizaje se centra en cuestiones relacionadas con los procesos y ambiente de aprendizaje. Se identificaron cuatro subcategorías: Entendimiento, Conocimiento, Facilidad y Motivación. El Entendimiento se refiere a la percepción de en qué medida el uso de TinkerPlots para o desarrollo de tareas amplió sus conocimientos sobre los conceptos estadísticos, les ayudó a entender mejor los conceptos anteriormente presentados en las clases y lo que se les pidió hacer en las tareas así como la posibilidad que han tenido de trabajar de forma autónoma en la finalización tareas. El Conocimiento se refiere a cómo lo uso de TinkerPlots facilitó a los estudiantes el aprendizaje de nuevos conceptos o procedimientos que no conocían antes. Facilidad se refiere a la percepción de los estudiantes de cómo TinkerPlots puede ser útil en su proceso de aprendizaje mediante la transformación de los ejercicios de una manera fácil y más perceptiva para ellos. La Motivación se refiere a la percepción de lo motivados que se sentían los estudiantes para participar en la resolución de las tareas y para mejorar su aprendizaje; "divertido" e "interesante" eran dos adjetivos que utilizaron para caracterizar estas clases.

De las cuatro preguntas planteadas forma negativa surgieron un total de cuatro categorías principales y doce subcategorías.

La categoría de Tecnología se refiere a las cuestiones que los estudiantes perciben como menos agradables en esta experiencia, específicamente en lo que se refiere al software TinkerPlots. Esta categoría se subdivide en cinco subcategorías: Manipulación, Instrumentalidad, Medidas, Limitaciones y Sin Desventajas. La Manipulación se refiere a la falta de dominio o familiaridad de los estudiantes con el nuevo software. Instrumentalidad se refiere a la incapacidad que los estudiantes parecían mostrar en esta experiencia y al uso de la tecnología como parte del proceso de aprendizaje y como contribución para mejorar sus conocimientos y 
habilidades. A subcategoría Medidas abarca la falta de apreciación de los estudiantes para el uso de TinkerPlots para calcular las medidas estadísticas específicas. Limitaciones considera la percepción de los estudiantes de TinkerPlots como un software restringido. Sin Desventajas denota el reconocimiento de la ausencia de desventajas o debilidades de los estudiantes en el uso de TinkerPlots para resolver las tareas.

La categoría de Aprendizaje se centra en cuestiones relacionadas con la disposición de los estudiantes para aprender o mejorar sus conocimientos, con dos subcategorías identificadas: Interés y Conocimiento. El Interés se refiere a la disposición de los estudiantes para aprender a lo largo de esta experiencia. El Conocimiento incluye la percepción de los estudiantes que ya conocían el contenido específico de la estadística.

La categoría Clase se refiere a las consecuencias desagradables percibidas de la metodología de enseñanza adoptada en esta experiencia. Se identificaron dos subcategorías: La Recolección De Datos y el Ambiente del aula. La Recolección de Datos incluye el malestar de los estudiantes con los asuntos directamente relacionados con la operación de recogida de datos. El Ambiente del Aula se refiere a la percepción de los estudiantes acerca de las condiciones físicas desagradables del aula.

La categoría Grupos respecta al trabajo del estudiante con sus compañeros en esta experiencia. Surgieron tres subcategorías: Relación, Trabajo y Orientación. Relación se refiere a la incomodidad que algunos estudiantes sintieron al trabajar con alguien con quien no tienen empatía o afinidad. La subcategoría Trabajo incluye la incapacidad de los estudiantes para organizar el trabajo con sus pares y desarrollar el apoyo mutuo. Orientación abarca las situaciones en las que los estudiantes no se centraron en el trabajo, debido a que el grupo era una fuente de perturbación.

\section{Resultados}

Después de desarrollar el análisis del contenido de las respuestas de todos los estudiantes en base a las categorías y subcategorías indicadas anteriormente, obtuvimos las Tablas 1 y 2 , donde se presenta un ejemplo de respuesta de un estudiante cualquiera de cada subcategoría con el fin de ilustrar su significado. Debemos indicar que algunos estudiantes respondieron de forma incompleta a las preguntas (p.e., "Sí” o "No" sin más explicaciones); estas respuestas no fueron incluidas en ninguna categoría o subcategoría con las que guar- 
daban relación, aunque sí fueron consideradas como respuestas válidas a la cantidad total de respuestas (N) de cada pregunta.

Tabla 1. Clasificación de la respuesta de los estudiantes a las cuatro preguntas planteadas en la forma positiva, por categorías y subcategorías

\begin{tabular}{|c|c|c|c|}
\hline Pregunta & Categoría & Subcategoría & Ejemplos \\
\hline \multirow{3}{*}{$\begin{array}{c}\text { 1. “¿En tu opi- } \\
\text { nión, el uso del } \\
\text { TinkerPlots para } \\
\text { desarrollar las } \\
\text { tareas facilitó tu } \\
\text { aprendizaje de la } \\
\text { estadística? Si es } \\
\text { así, explica por } \\
\text { qué." } \\
\text { (N=46) }\end{array}$} & $\begin{array}{l}\text { Clase } \\
(\mathrm{n}=4)\end{array}$ & $\begin{array}{c}\text { Novedad }(n=4) \\
\text { Grupos }(n=0)\end{array}$ & "Sí, porque era una manera diferente de aprender matemáticas." \\
\hline & $\begin{array}{c}\text { Tecnología } \\
(n=45)\end{array}$ & $\begin{array}{l}\text { Experiencia }(\mathrm{n}=11) \\
\text { Organización }(\mathrm{n}=7) \\
\text { Prático }(\mathrm{n}=8) \\
\text { Medidas }(\mathrm{n}=19)\end{array}$ & $\begin{array}{l}\text { "Sí, podemos aprender mejor usando TinkerPlots." } \\
\text { "Sí, porque es mucho más fácil de organizar todo." } \\
\text { "...Yo he preferido hacer los cálculos en el ordenador y podemos hacer gráficos más rápido que con la mano." } \\
\text { "Creo que sí, porque el trabajo fue conocer la mediana, media y modo.” }\end{array}$ \\
\hline & $\begin{array}{l}\text { Aprendizaje } \\
\quad(n=44)\end{array}$ & $\begin{array}{l}\text { Entendimiento }(\mathrm{n}=18) \\
\text { Conocimiento }(\mathrm{n}=12) \\
\text { Facilidade }(\mathrm{n}=10) \\
\text { Motivación }(\mathrm{n}=4)\end{array}$ & $\begin{array}{l}\text { "Sí, porque nunca he entendido diagramas de caja y por medio de este software fue capaz de hacerlo." } \\
\text { "Sí, yo no sabía cómo utilizar la estadística antes y con TinkerPlots he aprendido." } \\
\text { "Se ha facilitado en gran medida mi aprendizaje con los gráficos..." } \\
\text { "Sí, ya que fue muy divertido, aprendí rápidamiente." }\end{array}$ \\
\hline \multirow{3}{*}{$\begin{array}{l}\text { 2. “¿Cuáles fueron } \\
\text { las ventajas de } \\
\text { utilizar Tinker- } \\
\text { Plots en el clase?” } \\
\quad(\mathrm{N}=61)\end{array}$} & $\begin{array}{c}\text { Clase } \\
(\mathrm{n}=10)\end{array}$ & $\begin{array}{c}\text { Novedad }(\mathrm{n}=6) \\
\text { Grupos }(\mathrm{n}=4)\end{array}$ & $\begin{array}{l}\text { "... No teníamos lecciones orales, pero lecciones prácticas." } \\
\text { "Las ventajas fueron porque nosotros interactúamos más con nuestros colegas..." }\end{array}$ \\
\hline & $\begin{array}{l}\text { Tecnología } \\
(n=44)\end{array}$ & $\begin{array}{l}\text { Experiencia }(\mathrm{n}=16) \\
\text { Organización }(\mathrm{n}=1) \\
\text { Prático }(\mathrm{n}=10) \\
\text { Medidas }(\mathrm{n}=17)\end{array}$ & $\begin{array}{l}\text { "TinkerPlots es una manera práctica, fácil de estudiar matemáticas" } \\
\text { “... [El software] tiene todos los contenidos que hemos aprendido y porque es fácil acceder a todo." } \\
\text { “...Hemos sido capaces de representar los datos en cualquier gráfico rápidamente." } \\
\text { "...Hemos aprendido más acerca de gráficos, media y la mediana." }\end{array}$ \\
\hline & $\begin{array}{l}\text { Aprendizaje } \\
\quad(n=55)\end{array}$ & $\begin{array}{l}\text { Entendimiento }(\mathrm{n}=16) \\
\text { Conocimiento }(\mathrm{n}=11) \\
\text { Facilidade }(\mathrm{n}=20) \\
\text { Motivación }(\mathrm{n}=8)\end{array}$ & $\begin{array}{l}\text { "...Empecé a mejorar mi aprendizaje con gráficos." } \\
\text { “...Hemos aprendido cómo realizar los gráficos de diferentes maneras." } \\
\text { "...El software hay facilitado la resolución de las tareas." } \\
\text { "El TinkerPlots hizo el aprendizaje estadístico más interesante y divertido." }\end{array}$ \\
\hline \multirow{3}{*}{$\begin{array}{l}\text { 3. “¿Qué has } \\
\text { disfrutado más al } \\
\text { trabajar con Tin- } \\
\text { kerPlots?" } \\
(\mathrm{N}=61)\end{array}$} & $\begin{array}{l}\text { Clase } \\
(\mathrm{n}=4)\end{array}$ & $\begin{array}{l}\text { Novedad }(\mathrm{n}=4) \\
\text { Grupos }(\mathrm{n}=0)\end{array}$ & "Me gustó todo porque para mí fue una experiencia nueva y pegadiza." \\
\hline & $\begin{array}{l}\text { Tecnología } \\
(n=96)\end{array}$ & $\begin{array}{l}\text { Experiencia }(\mathrm{n}=19) \\
\text { Organización }(\mathrm{n}=3) \\
\text { Prático }(\mathrm{n}=35) \\
\text { Medidas }(\mathrm{n}=39) \\
\end{array}$ & $\begin{array}{l}\text { "...Trabajar las matemáticas en el ordenador, ya que es algo nuevo ya que trabajamos más con el papel." } \\
\text { "Lo que más me gustó fue ver cómo las bolas se organizaban." } \\
\text { "El TinkerPlots sólo nos dava los resultados que queríamos." } \\
\text { "Lo que más me gustó fue trabajar con la media y la mediana." }\end{array}$ \\
\hline & $\begin{array}{l}\text { Aprendizaje } \\
\quad(\mathrm{n}=9)\end{array}$ & $\begin{array}{l}\text { Entendimiento }(\mathrm{n}=2) \\
\text { Conocimiento }(\mathrm{n}=1) \\
\text { Facilidade }(\mathrm{n}=3) \\
\text { Motivación }(\mathrm{n}=3)\end{array}$ & $\begin{array}{l}\text { “...Con el TinkerPlots era mucho más fácil entender lo que estábamos llamados a realizar.” } \\
\text { "Lo que más me gustó fué descubrir el gráfico de caja.” } \\
\text { "Me gustó mucho trabajar con el TinkerPlots porque era mucho más fácil tener que hacer cálculos.” } \\
\text { “...Es una buena manera para que nosotros tengamos diversión mientras llevamos a cabo ejercicios de matemáticas." }\end{array}$ \\
\hline 4. “'Te ayudó el & $\begin{array}{c}\text { Clase } \\
(\mathrm{n}=52)\end{array}$ & $\begin{array}{l}\text { Novedad }(\mathrm{n}=0) \\
\text { Grupos }(\mathrm{n}=52)\end{array}$ & $\begin{array}{l}\text { "Sí, porque éramos un grupo, estábamos más para discutir ideas y opiniones"; "Sí, porque mi grupo podría saber } \\
\text { cosas que yo no había hecho"; "Sí, porque como equipo podemos lograr cosas que por separado no hemos podido"; }\end{array}$ \\
\hline
\end{tabular}




\begin{tabular}{|c|c|c|c|}
\hline \multirow{3}{*}{$\begin{array}{l}\text { trabajo en grupo } \\
\text { para desarrollar } \\
\text { las tareas? Expli- } \\
\text { ca" } \\
(\mathrm{N}=56)\end{array}$} & & & $\begin{array}{l}\text { "Me ayudó mucho, si no sabía cosas le pregunté a mi colega y ella me ayudó, y yo la ayudé"; "Sí, porque sólo tal vez } \\
\text { no habría sido capaz de hacer ni la mitad de lo que he hecho." }\end{array}$ \\
\hline & $\begin{array}{c}\text { Tecnología } \\
(\mathrm{n}=0)\end{array}$ & $\begin{array}{l}\text { Experiencia }(\mathrm{n}=0) \\
\text { Organización }(\mathrm{n}=0) \\
\text { Prático }(\mathrm{n}=0) \\
\text { Medidas }(\mathrm{n}=0)\end{array}$ & \\
\hline & $\begin{array}{c}\text { Aprendizaje } \\
(n=54)\end{array}$ & $\begin{array}{l}\text { Entendimiento }(\mathrm{n}=30) \\
\text { Conocimiento }(\mathrm{n}=6) \\
\text { Facilidade }(\mathrm{n}=15) \\
\text { Motivación }(\mathrm{n}=3)\end{array}$ & $\begin{array}{l}\text { "Sí, ya estamos más [personas] a pensar. Podríamos pensar más y mejor organización de las ideas.” } \\
\text { "Sí, porque hemos sido capaces de aprender con nuestros compañeros lo que no sabíamos." } \\
\text { "Sí, porque dos cabezas piensan mejor que una, por lo tanto, era mucho más fácil de llevar a cabo las tareas." } \\
\text { “... Me ayudó mucho para convertir las lecciones en algo más divertido.” }\end{array}$ \\
\hline
\end{tabular}

Tabla 2. Clasificación de la respuesta de los estudiantes a las cuatro preguntas planteadas en la forma negaiva, por categorías y subcategorías

\begin{tabular}{|c|c|c|c|}
\hline Pregunta & Categoría & Subcategoría & Ejemplos \\
\hline \multirow{2}{*}{$\begin{array}{l}\text { 5. “¿En tu opinión, el uso de } \\
\text { TinkerPlots para desarrollar } \\
\text { las tareas no facilitó el } \\
\text { aprendizaje estadística? Si es } \\
\text { así, explica por qué.” } \\
(\mathrm{N}=12)\end{array}$} & $\begin{array}{l}\text { Tecnología } \\
\quad(n=3)\end{array}$ & Manipulación $(n=3)$ & "No facilitó ya que había opciones difíciles de encontrar." \\
\hline & $\begin{array}{l}\text { Aprendizaje } \\
(\mathrm{n}=3)\end{array}$ & $\begin{array}{c}\text { Interés }(\mathrm{n}=1) \\
\text { Conocimiento }(\mathrm{n}=2)\end{array}$ & $\begin{array}{l}\text { "No, yo estaba sin preocupaciones sobre el trabajo que hay que hacer." } \\
\text { "No, porque no tenía dudas en las estadísticas." }\end{array}$ \\
\hline \multirow[t]{2}{*}{$\begin{array}{l}\text { 6. “¿Cuáles fueron las des- } \\
\text { ventajas de usar TinkerPlots } \\
\text { en el clase?” }(\mathrm{N}=41)\end{array}$} & $\begin{array}{l}\text { Tecnología } \\
(\mathrm{n}=38)\end{array}$ & $\begin{array}{l}\text { Manipulación }(n=12) \\
\text { Instrumentalidad }(n=3) \\
\text { Sin Desventajas }(n=23)\end{array}$ & $\begin{array}{l}\text { "...Vamos a necesitar más lecciones [utilizando TinkerPlots] para aprender a trabajar en él..." } \\
\text { "La desventaja era que no nos preparamos para el examen final." } \\
\text { "No tengo nada a decir sobre las desventajas porque no había ninguna." }\end{array}$ \\
\hline & $\begin{array}{l}\text { Clase } \\
(\mathrm{n}=3)\end{array}$ & $\begin{array}{l}\text { Recolección de Datos }(\mathrm{n}=1) \\
\text { Ambiente }(\mathrm{n}=2)\end{array}$ & $\begin{array}{l}\text { "Las desventajas eran guardar los datos manuales." } \\
\text { "Había demasiado ruido." }\end{array}$ \\
\hline \multirow[t]{2}{*}{$\begin{array}{l}\text { 7. "¿Qué han disfrutado } \\
\text { menos en el trabajo con } \\
\text { TinkerPlots?" } \\
\text { (N=37) }\end{array}$} & $\begin{array}{l}\text { Tecnología } \\
(\mathrm{n}=29)\end{array}$ & $\begin{array}{l}\text { Manipulación }(\mathrm{n}=3) \\
\text { Medidas }(\mathrm{n}=17) \\
\text { Limitaciones }(\mathrm{n}=1) \\
\text { Sin Desventajas }(\mathrm{n}=8)\end{array}$ & $\begin{array}{l}\text { "Tenía mucha dificultad para trabajar con el software desde el princípio." } \\
\text { "No me gusta la forma que tenemos de ver la mediana." } \\
\text { "Lo que menos me gustó fue que [el TinkerPlots] no tenía más cosas..." } \\
\text { "No hubo nada que me ha disgustado." }\end{array}$ \\
\hline & Clase $(n=8)$ & Recolección de Datos $(n=8)$ & "Lo que menos me gustó fue la necesidad de grabar los archivos a todo el tiempo." \\
\hline $\begin{array}{l}\text { 8. "¿El trabajo en grupo no } \\
\text { te ayudó a desarrollar las } \\
\text { tareas? Explica." } \\
(\mathrm{N}=10)\end{array}$ & $\begin{array}{l}\text { Grupos } \\
(\mathrm{n}=10)\end{array}$ & $\begin{array}{l}\text { Relación }(\mathrm{n}=4) \\
\text { Trabajo }(\mathrm{n}=4) \\
\text { Orientación }(\mathrm{n}=2)\end{array}$ & $\begin{array}{l}\text { "...Me gusta trabajar con la gente que más confío y com quien tengo una mejor relación." } \\
\text { "Mi colega simplemente escribó en el ordenador. Por desgracia, tuve que hacer solo todo el restante } \\
\text { [trabajo]." } \\
\text { "...En la primera tarea hemos empezado a hablar mucho y no terminamos la tarea." }\end{array}$ \\
\hline
\end{tabular}


El análisis de la pregunta $1(\mathrm{~N}=46)$ muestra que los estudiantes valoran muy positivamente el uso de las TIC para la resolución de las tareas en estas aulas (Tecnología; n=45). La posibilidad de utilizar el TinkerPlots para explorar las Medidas estadísticas específicas (n=19) fue el aspecto que mencionan más, seguidas por la oportunidad de llegar a conocer el software TinkerPlots (Experiencia; n=11). También valoraron el carácter Práctico de las TIC (n=8) y reconocen las funciones de TinkerPlots para organizar los datos y la información estadística, en general (Organización; n=7). También hay un número significativo de referencias que expresan su reconocimiento de los efectos de TinkerPlots en sus procesos de aprendizaje en la estadística (Aprendizaje; $\mathrm{n}=44$ ), poniendo de relieve cómo esta experiencia les ayudó a entender los conceptos estadísticos que han aprendido previamente pero que no estaban totalmente entendidos, y de interpretar lo que les fue demandado por las preguntas que se ses propusieron (Entendimiento; $\mathrm{n}=18$ ). Algunos estudiantes también percibieron que esta experiencia les permitió mejorar sus conocimientos de estadística (Conocimiento; $\mathrm{n}=12$ ).

En cuanto a la pregunta 2, casi todos los estudiantes $(\mathrm{N}=61)$ identifican las ventajas de utilizar el ordenador en estas aulas. La mayor parte de las referencias han hecho énfasis en el uso de las TIC como valiosa y útil para su aprendizaje en la estadística (Aprendizaje; $\mathrm{n}=55$ ). Facilidad era el aspecto de esa categoría que mencionaron la mayoría de los estudiantes $(n=20)$, seguido de Entendimiento $(n=16)$. En cuanto a la capacidad de resiliencia matemática es importante notar que si analizamos conjuntamente las subcategorías Entendimiento y Conocimiento $(\mathrm{n}=27)$ abarcan la mayoría de las referencias de los estudiantes en relación con las ventajas de utilizar el software en el aula dentro de la categoría de Aprendizaje. En lo que respecta específicamente a la inclusión de las TIC en estas aulas (Tecnología; n=44), los estudiantes pusieron de relieve sus ventajas con respecto a la posibilidad de explorar diferentes medidas de tendencia central, tales como diagramas de caja, media y mediana (Medidas; $\mathrm{n}=17)$. Los estudiantes también perciben ésto como una Experiencia $(\mathrm{n}=16)$ inusual y no convencional que les permitió aprender la estadística en un entorno de aprendizaje diferente.

En lo que se refiere la pregunta $3(\mathrm{~N}=61)$, los estudiantes destacaron claramente el uso del software (Tecnología; n=96) como el aspecto más agradable de la experiencia. Señalaron las medidas de tendencia central como el aspecto con el que más disfrutaron trabajar (Medidas; $\mathrm{n}=39) \mathrm{y}$, en segundo lugar, expresan su preferencia por las características del software que les permitieron realizar más en menos tiempo y poner en práctica sus conocimientos teóricos (Prático; $\mathrm{n}=35)$. 
En lo que se refiere a la pregunta $4(\mathrm{~N}=54)$, los estudiantes han reconocido ampliamente el impacto positivo del trabajo en grupos en su proceso de Aprendizaje $(\mathrm{n}=54)$. La Comprensión $(\mathrm{n}=30)$ fue el aspecto más mencionado por los estudiantes, ya que perciben que las ideas del otro facilitaron profundizar en la comprensión y mejorar su entendimimento de los conceptos estadísticos. Los estudiantes también destacaron el cambio de entorno de Clase $(n=52)$, específicamente en cuanto a la posibilidad de trabajar con sus compañeros, y valuaron compartir las ideas entre sí (Grupos; $\mathrm{n}=52$ ). Los estudiantes no mencionaron la tecnología en relación con el trabajo en grupo.

En cuanto a las preguntas planteadas en forma negativa, al responder a la pregunta 5 hay algunos estudiantes $(\mathrm{N}=12)$ que no consideran que el uso de TinkerPlots facilitara su aprendizaje en estadística. Un número residual de estudiantes tuvo la percepción de TinkerPlots (Tecnología; $\mathrm{n}=3$ ) como una herramienta difícil de manejar (Manipulación; $\mathrm{n}=3$ ) y sugirieron que era un obstáculo para su desempeño.

Por lo que respecta a la pregunta $6(\mathrm{~N}=41)$, dentro de la categoría de Tecnología $(n=38)$ la mayoría de los estudiantes expresa la ausencia de desventajas en el uso de las TIC en estas aulas (Sin Desventajas; $\mathrm{n}=23$ ), mientras que otros señalaron la dificultad que se encontraron al utilizar TinkerPlots, alegando la necesidad de familiarizarse con esta herramienta con más profundidad (Manipulación; $\mathrm{n}=12$ ).

En la pregunta $7(\mathrm{~N}=37)$, los estudiantes comentaron aspectos específicos del software que les parecía haber sido menos agradables para ellos (Tecnología; $\mathrm{n}=29$ ), especialmente con referencia a determinadas medidas estadísticas o procedimientos (Medidas; $\mathrm{n}=17$ ), aunque otros no identificaron ningún aspecto menos agradable en cuanto a su uso (Sin Desventajas; $\mathrm{n}=8)$.

Por último, en cuanto a la pregunta $8(\mathrm{~N}=10)$, un pequeño número de estudiantes percibió la experiencia de trabajar con sus compañeros como incómodas (Grupos; $\mathrm{n}=10$ ), principalmente por razones de afinidad (Relación; $\mathrm{n}=4$ ) o por una falta de sentido de Trabajo cooperativo $(n=4)$. 


\section{Discusión}

Los resultados descritos anteriormente ilustran aspectos importantes en relación con la noción de la resiliencia matemática que ha sido adoptada en esta investigación. Los estudiantes informaron que estas experiencias les permiten aprender nuevos contenidos y tener una comprensión más profunda de los contenidos aprendidos con anterioridad, que tienen la idea subyacente de que creen que pueden mejorar su proceso de aprendizaje y calcanzar mejores resultados en matemáticas. Los estudiantes también pusieron de relieve la importancia de sus compañeros en esta experiencia, el reconocimiento de la ayuda mutua y el espíritu de equipo como aspctos esenciales para el éxito de en la finalización de la tarea, lo cual es un elemento importante cuando se caracteriza la resiliencia matemática (Lee \& Johnston-Wilder, 2013). La importancia del trabajo en equipo percibida por los estudiantes así como la ayuda mutua que dieron y reciben de sus compañeros, parece ser una fuente de comportamientos de perseverancia para los estudiantes, que los hacen avanzar en el proceso de aprendizaje, con el apoyo de sus colegas, cuando se enfrentan a dificultades en ese proceso.

A pesar de estos resultados positivos, no emergen de los estudiantes respuestas respecto a su comprensión de la instrumentalidad del contenido de las matemáticas fuera de la clase. Ya que esta premisa no se les preguntó de forma intencionada, no podríamos asumir que hay una falta de esta comprensión; a su vez, ya que las tareas desarrolladas por los estudiantes se basaron en principios SRLE, especialmente la utilización de los datos reales, podemos suponer que los estudiantes son conscientes de la relación entre la actividad que han desarrollado y el contexto académico exterior. No obstante, los profesores de estas aulas hacen hincapié en la importancia de que los estudiantes trabajen con datos reales en estas tareas (Oliveira \& Henriques, 2014).

Los resultados presentados anteriormente ilustran aspectos importantes manifestados por los estudiantes sobre el uso de las TIC en la clase de matemáticas. Uno de los más notables es que consideran que las TIC les permiten realizar las tareas de forma fácil y rápida. De acuerdo a su opinión, los estudiantes fueron capaces de acceder de forma sencilla a una gran cantidad de datos y organizarlos mejor y hacer diversos tipos de representaciones gráficas y cálculos estadísticos para medidas específicas. En consecuencia, podríamos suponer que perciben los procesos de aprendizaje creados por la inclusión de las TIC en sus aulas como más fáciles para ellos, lo que significa que sentían que podían tener un mejor desempeño y mejorar 
su aprendizaje en un entorno SRLE. Al mismo tiempo, los estudiantes reportaron esta experiencia como diferente y agradable, a la vez que motivadora para desarrollar las actividades propuestas en clase.

Aunque la mayoría de los estudiantes señaló resultados positivos de esta experiencia, algunos reportaron sentimientos de angustia mientras que otros de indiferencia. Por un lado, la falta de habilidades técnicas por los estudiantes para dominar las herramientas de TinkerPlots inhibe su participación activa en las tareas y genera sentimientos negativos acerca de sus capacidades para mejorar el aprendizaje. Por otra parte, algunos estudiantes no percibierron las tareas como parte del contenido de su aprendizaje matemático y no ven esta experiencia como clases de matemáticas. A pesar de la cantidad residual de los estudiantes que señalan estas dos percepciones, estos hallazgos sugieren la importancia de asegurar que todos los estudiantes tengan las habilidades necesarias para dominar las herramientas TIC específicas en el aula, dándoles un plazo de adaptación razonable; también podríamos suponer que, para algunos estudiantes, los profesores podrían no haber dado el apoyo que necesitan para desarrollar las tareas de una manera segura y eficaz. Estos resultados son consistentes con los resultados de investigaciones anteriores en cuanto a la voz de los estudiantes acerca de las experiencias de las TIC (p.e., Deaney et al., 2003) y corroboran la idea de que las TIC podrían mejorar los resultados educativos (Livingstone, 2012).

\section{Conclusiones}

El objetivo principal de esta investigación fue estudiar cómo los estudiantes que asisten a escuelas de la zona suburbana de la capital portuguesa son capaces de reconocer y exhibir características de resiliencia matemática después de resolver tareas utilizando el software TinkerPlots en el tema de la estadística. Aunque no podemos afirmar que el uso de las TIC en la clase de matemáticas desarrolla la capacidad de resiliencia matemática de los estudiantes, podemos afirmar que estos estudiantes portugueses exhiben algunas características que la literatura especializada describe como típica para los estudiantes matemáticamente resilientes (Lee \& Johnston-Wilder, 2013). Mediante el uso de la tecnología adecuada para ayudar al proceso de enseñanza-aprendizaje y con el apoyo de sus compañeros y profesores, los estudiantes fueron capaces de resolver tareas con objetivos de aprendizaje complejos.

Puesto que las matemáticas son percibidas por los estudiantes como una de las materias escolares más exigentes y difíciles, se hace imperativo el desarrollo de estrategias en las 
escuelas que desarrollen su confianza para mejorar su aprendizaje matemático y el rendimiento académico. Los profesores y estudiantes son los principales protagonistas del proceso de enseñanza-aprendizaje y por eso es importante crear la conciencia de los profesores de su papel en la promoción de la resiliencia matemática. Para desarrollar y promover la resiliencia matemática de una manera eficaz y reflexiva se hace necesario que los profesores comprendan su importancia en el logro académico, específicamente en las matemáticas, y sean capaces de identificar oportunidades para trabajar la resiliencia con sus estudiantes en el contexto de vida cotidiana de sus aulas. Es prioritario que los profesores promueven intencionadamente y regularmente un comportamiento flexible en sus estudiantes que les permita comprender las dificultades que enfrentan y cómo pueden superarlas; por eso, los estudiantes necesitan sentir que pueden tener el apoyo que necesitan y que el esfuerzo que ponen en la actividad matemática vale la pena.

Teniendo en cuenta que los estudiantes son los principales actores del proceso de aprendizaje, en este estudio nos propusimos posibilitar que expresaran sus puntos de vista sobre los temas que son percibidos como importantes por ellos para su compromiso con el aprendizaje y progreso. Al permitir a los estudiantes expresarse, los profesores pueden obtener nuevos conocimientos sobre cómo los estudiantes pueden mejorar su aprendizaje y sobre su compromiso con los asuntos escolares. Esto es particularmente significativo con los estudiantes con resultados más bajos, ya se les ofrece la oportunidad y el estímulo para reflexionar sobre su propio proceso de aprendizaje (Flutter, 2007). Este nuevo enfoque es contrario a las prácticas convencionales restrictivas (Nardi \& Steward, 2003) que tienen actualmente lugar en las clases en las que el profesor tiene un papel principal a tiempo completo y muestra los conceptos que hay que aprender, seguidos por la resolución de ejercicios por los estudiantes. Los nuevos enfoques, como el SRLE que se describe en este articulo, requiere el cambio de una mentalidad para la cual toda la comunidad escolar debe ser sensibilizada (Yeager \& Dweck, 2012).

Los psicólogos en las escuelas podrían contribuir a una toma de conciencia de los profesores y los padres de la importancia de promover y ayudar a los estudiantes a desarrollar conductas resilientes en las matemáticas y en otras cuestiones curriculares, así como en temas extraescolares. Como dice Yeager y Dweck (2012), “cuando hacemos hincapié en el potencial de las personas para cambiar, preparamos a nuestros estudiantes para hacer frente a desafíos de la vida de forma resiliente" (p. 312). 


\section{Limitaciones}

Aunque este estudio nos permite comenzar a concebir cómo el uso de las TIC en las clases puede promover la resiliencia matemática de los estudiantes en determinadas circunstancias, también hay algunas limitaciones. En primer lugar, ya que este estudio forma parte de un proyecto de investigación más amplio que pretende desarrollar la cultura estadística de los estudiantes, las tareas desarrolladas en este trabajo se centraron en el ámbito estadístico y no en las matemáticas en general. Por lo tanto, ya que el software está orientado sólo para el tema de la estadística, se necesitan más estudios con otros programas dirigidos a diferentes contenidos en matemáticas. También identificamos limitaciones en el instrumento utilizado para la recolección de datos con el fin de comprender plenamente todos los elementos que deben considerarse en la capacidad de resiliencia matemática. En un estudio adicional, este método también podría ser complementado con diferentes datos de la clase. Por último, para mejorar significativamente la capacidad de resiliencia matemática de los estudiantes sería necesario ampliar la experiencia por un período de tiempo más largo.

\section{Expresiones de gratitud}

Ref del proyecto PTDC/CPE-CED/117933/2010. El desarrollo de la literacia estadística: El aprendizaje del estudiante y la formación del profesorado. Financiado por FCT - Fundación para la Ciencia y la Tecnología, de Portugal (2012-2015). Expresamos nuestro sincero agradecimento a Ana Caballero por su ayuda en la revisión del texto en español.

\section{Referencias}

Bardin, L. (2014). Análise de conteúdo [Análisis de Contenido]. Lisboa, Portugal: Edições 70, Lda.

Ben-Zvi, D. (2006). Scaffolding students' informal inference and argumentation. En A. Rossman \& B. Chance (Eds.), Atas de la Seventh International Conference on Teaching Statistics (ICOTS 7). Obtenido de http://blog.apastyle.org/apastyle/2012/08/howto-cite-materials-from-meetings-and-symposia.html

Cobb, P., \& McClain, K. (2004). Principles of instructional design for supporting the development of students' statistical reasoning. En D. Ben-Zvi \& J. Garfield (Eds.), The challenge of developing statistical literacy, reasoning, and thinking (pp. 375-396). Dordrecht, The Netherlands: Kluwer Academic Publishers.

Deaney, R., Ruthven, K., \& Hennessy, S. (2003). Pupil perspectives on the contribution of Information and Communication Technology to teaching and learning in the Secondary school. Research Papers in Education, 18(2), 141-165. doi: 10.1080/026715232000081913. 
Dweck, C. (2000). Self theories: Their role in motivation, personality and development. Lillington: Psychology Press, Taylor \& Francis.

Fergus, S., \& Zimmerman, M. A. (2005). Adolescent resilience: A framework for understanding healthy development in the face of risk. Annual Review Public Health, 26, 399419. doi: 10.1146/annurev.publhealth.26.021304.144357.

Fitzallen, N. (2007). Evaluating data analysis software: The case of TinkerPlots. Australian Primary Mathematics Classroom, 12(1), 23-38. Obtenido de http://files.eric.ed.gov/fulltext/EJ793972.pdf

Fitzallen, N., \& Watson, J. (2010). Developing statistical reasoning facilitated by Tinkerplots. En C. Reading (Ed.), Atas de la Eighth International Conference on Teaching Statistics (ICOTS $\quad$ Obtenido de https://www.stat.auckland.ac.nz/ iase/publications/icots8/ICOTS8_8A4_FITZALLEN .pdf

Flutter, J. (2007). Teacher development and pupil voice. The Curriculum Journal, 18(3), 343354. doi: 10.1080/09585170701589983.

Garfield, J., \& Ben-Zvi, D. (2009). Helping students develop statistical reasoning: Implementing a statistical reasoning learning environment. Teaching Statistics, 31(3), 72-77. doi: 10.1111/j.1467-9639.2009.00363.x

Garfield, J., delMas, R., \& Zieffler, A. (2012). Developing statistical modelers and thinkers in an introductory, tertiary-level statistics course. ZDM Mathematics Education, 44, 883898. doi: 10.1007/s11858-012-0447-5.

González-Torres, M., \& Artuch-Ragarde, R. (2014). Resilience and coping strategy profiles at university: Contextual and demographic variables. Electronic Journal of Research in Educational Psychology, 12(3), 621-648. doi: 10.14204/ejrep.34.14032.

Henriques, A., \& Oliveira H. (2016). Students' informal inference when exploring a statistical investigation. En K. Krainer \& N. Vondrová (Eds.), Atas de la Ninth Congress of European Research in Mathematics education (pp. 685-691). Prague, Czech Republic: Faculty of Education, Charles Univeristy in Prague.

Johnston-Wilder, S., \& Lee, C. (2010). Mathematical resilience. Mathematics Teaching, 218, $38-41$.

Kent, N., \& Facer, K. (2004). Different worlds? A comparison of young people's home and school ICT use. Journal of Computer Assisted Learning, 20(6), 440-455. doi: 10.1111/j.1365-2729.2004.00102.x.

Konold, C., \& Miller, C.D. (2005). TinkerPlots: Dynamic Data Exploration ${ }^{T M}$ (versión 1.0) [Software de ordenador]. Emeryville, CA: Key Curriculum Press.

Lee, C., \& Johnston-Wilder, S. (2013). Learning mathematics - letting the pupils have their say. Educational Studies in Mathematics, 83(2), 163-180. doi: 10.1007/s10649-0129445-3. 
Livingstone, S. (2012). Critical reflections on the benefits of ICT in education. Oxford Review of Education, 38(1), 9-24. doi: 10.1080/03054985.2011.577938.

Lugalia, M., Johnston-Wilder, S., \& Goodall, J. (2013). The role of ICT in developing mathematical resilience in learners. Atas de la Seventh International Technology, Education and Development Conference (INTED 7) (pp. 4096-4105). Valencia, España: IATED. ISBN: 978-84-616-2661-8.

Luthar, S. S. (2007). Resilience in development: A synthesis of research across five decades. En D. Cicchetti \& D.J. Cohen (Eds.). Developmental Psychopathology. Volume Three: Risk, Disorder, and Adaptation (2 ${ }^{\text {nd }}$ ed.) (pp.739-795.). Hoboken, NJ, US: John Wiley \& Sons Inc.

Makar, K., Bakker, A., \& Ben-Zvi, D. (2011). The reasoning behind informal statistical inference. Mathematical Thinking and Learning, 13(1-2), 152-173. doi: 10.1080/10986065.2011.538301.

Moore, D. S. (1997). New pedagogy and new content: The case of statistics. International Statistical Review, 65(2), 123-137. doi: 10.1111/j.1751-5823.1997.tb0039.x.

Nardi, E., \& Steward, S. (2003). Is Mathematics T.I.R.E.D.? A profile of quiet disaffection in the secondary mathematics classroom. British Educational Research Journal, 29(3), 345-367. doi: 10.1080/0141192031000155999.

Oliveira, H., \& Henriques A. (2014). Promover o raciocínio estatístico no ensino básico recorrendo à tecnologia: Um projeto de investigação e desenvolvimento [Promover el razonamiento estadístico en la educación básica mediante la tecnología: Un proyecto de investigación y desarrollo]. Boletim da Sociedade Portuguesa de Estatística, Outono, 23-31.

Rivera, H., \& Waxman, H. (2011). Resilience and nonresilient Hispanic English language learners' attitudes towards their classroom learning environment in mathematics. Journal of Education for Students Placed at Risk, 16(3), 185-200. doi: 10.1080/10824669.2011.585100.

Sutherland, R., Armstrong, V., Barnes, S., Brawn, R., Breeze, N., Gall, M., Matthewman, S., Olivero, F., Taylor, A., Triggs, P., Wishart, J., \& John, P. (2004). Transforming teaching and learning: Embedding ICT into everyday classroom practices. Journal of Computer Assisted Learning, 20(6), 413-425. doi: 10.1111/j.1365-2729.2004.00104.x.

Watson, J. M. (2012). Resampling with TinkerPlots. Teaching Statistics, 35(1), 32-36. doi: 10.1111/j.1467-9639.2012.00511.x.

Yeager, D., \& Dweck, C. (2012). Mindsets that promote resilience: When students believe that personal characteristics can be developed. Educational Psychologist, 47(4), 302314. doi: 10.1080/00461520.2012.722805. 